Check for updates

Cite this: RSC Adv., 2017, 7, 53157

Received 9th October 2017

Accepted 11th November 2017

DOI: 10.1039/c7ra11102a

rsc.li/rsc-advances

\title{
Controllable fabrication of Ag-nanoplate- decorated PAN-nanopillar arrays and their application in surface-enhanced Raman scattering $†$
}

\author{
Zhongbo Li, (D) *ab Zhaofang Du, ${ }^{* a}$ Kexi Sun, ${ }^{c}$ Xuan He ${ }^{d}$ and Bensong Chen*b
}

We report a facile and low-cost approach for fabrication of large-scale surface-enhanced Raman scattering (SERS) active substrates composed of Ag-nanoplates (Ag-NPs) decorated polyacrylonitrile nanopillar (denoted as Ag-NPs@PAN-nanopillar) arrays, via a consecutive process of molding PAN-nanopillar arrays, sputtering Au-nanoparticles onto the PAN-nanopillar arrays as a conducting layer, and decorating the PAN-nanopillars with Ag-nanoplates. The Ag-nanoplate distribution density on the PAN-nanopillars can be tailored by tuning the concentration of citric acid and the Ag-deposition duration, and high SERS sensitivity can thus be achieved by optimizing the density of Ag-nanoplates. The adjacent Ag-nanoplates induced "hot spots" are densely and uniformly distributed in the three dimensional (3D) space around the PAN-nanopillar arrays, and thus Ag-NPs@PAN-nanopillar arrays generated sensitive and homogenous SERS signals when using rhodamine $6 \mathrm{G}$ as a probed molecule. Using the Ag-NPs@PAN-nanopillar arrays as SERS substrates, $10^{-7} \mathrm{M}$ methyl parathion (organophosphorus insecticide) and $10^{-6} \mathrm{M} \mathrm{PCB}-77$ (one congener of polychlorinated biphenyls belonging to persistent organic pollutants) are identified. Therefore the SERS-active substrates have potential in SERS-based rapid detection of environmental organic pollutants.

\section{Introduction}

Surface-enhanced Raman scattering (SERS) has been developed as one of the most promising ultra-sensitive real time analytical techniques, which has led to major breakthroughs in a number of areas, including biological chemistry, environmental monitoring, and sensor applications. ${ }^{\mathbf{1 - 8}}$ One of the key issues for SERS-based ultra-trace analysis is rational design and fabrication of SERS substrates with high sensitivity and good signal reproducibility. ${ }^{\mathbf{9} 10}$ It is commonly accepted that SERS enhancement is largely due to the amplification of the local electromagnetic (EM) fields owing to the excitation of surface-plasmon resonance within the so-called "hot spots" located in the nanogaps, ${ }^{11,12}$ and around the sharp nanoedges ${ }^{13,14}$ and nanotips of the noble metal nanostructures. ${ }^{15,16}$ Therefore, a great deal of SERS research has been devoted to the optimization of noble

${ }^{a}$ College of Light-Textile Engineering and Art, Anhui Agricultural University, Hefei 230036, China.E-mail: zbli@ahau.edu.cn

${ }^{b}$ Key Laboratory of Materials Physics, Key Laboratory of Nanomaterials and Nanotechnology, Institute of Solid State Physics, Chinese Academy of Sciences, Hefei, Anhui 230031, China

${ }^{c}$ College of Physics and Electronic Information, Luoyang Normal University, Luoyang 471022, China

${ }^{d}$ Institute of Chemical Materials, China Academy of Engineering Physics, Mianyang 621900, China

$\dagger$ Electronic supplementary information (ESI) available. See DOI: 10.1039/c7ra11102a metal (particularly Ag or Au) SERS substrates with high density of uniformly distributed hot spots within diverse nanostructured architectures, such as zero dimensional (0D) single-particles, ${ }^{17}$ one dimensional (1D) metallic nanoparticle chains, ${ }^{18}$ two dimensional (2D) self-assembled metallic nanoparticle arrays ${ }^{19}$ and three dimensional (3D) noble-metal/semiconductor composite micro/nanostructures. ${ }^{20-25}$ Among them, SERS-active substrates based on the 3D ordered composite micro/ nanostructures have attracted ever-increasing attention, as "3D” SERS substrates can achieve high density of 3D electromagnetic "hot spots" and offer large surface areas which are beneficial to the adsorption of target analytes. ${ }^{\mathbf{1 4 , 2 6 , 2 7}}$ In addition, the ordered "3D" SERS substrates can ensure better reproducibility and reliability of SERS signals compared to the dispersed noble-metal colloidal nanoparticles. ${ }^{28}$

To date, a large number of "3D" SERS substrates have been achieved via a broad variety of routes. One universal strategy to develop 3D SERS substrates is decorating plasmonic nanostructures $(\mathrm{Ag}, \mathrm{Au})$ onto ordered 3D-frameworks such as carbon nanotube arrays, ${ }^{23}$ bioscaffold arrays, ${ }^{29}$ glass nanopillar arrays, ${ }^{26}$ and semiconductors ( $\mathrm{Si}, \mathrm{Ge}, \mathrm{ZnO}$, and $\mathrm{TiO}_{2}$ ) nanostructure arrays. ${ }^{30-32}$ Therefore, three types of hot spots can be achieved in the $3 \mathrm{D}$ ordered hybrid nanostructure arrays, ${ }^{26,28}$ i.e., the first one on the top ends of the individual building blocks of $3 \mathrm{D}$ framework, the second one on the sidewalls of individual building blocks of 3D-framework nanoblock, and the third one between the neighboring building blocks of 3D-framework 
nanoblock. On the other hand, Ag nanoplates have attracted researchers' attention due to their unique sharp edges, which can provide extraordinary localized surface plasmon resonance (LSPR) compared to the traditional Ag nanoparticles (NPs) and can be widely employed as plasmonic nanoscale building blocks for constructing effective SERS substrates. ${ }^{14,33,34}$ Therefore, if the $\mathrm{Ag}$ nanoplates are assembled into the 3D ordered nanoarchitectured frameworks, high density "hot spots" could be achieved in the gaps between the neighboring nanoplates, and thus 3D hierarchical SERS-substrates with high sensitivity and good signal reproducibility could be expected.

Herein, we present a simple and low-cost method for fabricating large-area SERS-active substrates made of vertically aligned PAN-nanopillar arrays decorated with high density Ag nanoplates via a successive process, as shown schematically in Fig. 1. Firstly, large-area highly ordered vertically aligned PANnanopillar arrays were facilely prepared. Subsequently, Au nanoparticles are sputtered onto the PAN-nanopillars to service as a conducting layer and also provide nucleation sites for $\mathrm{Ag}$ nanostructures to grow. Finally, Ag nanoplates are assembled onto the Au-nanoparticles decorated PAN-nanopillars via simple galvanic cell reaction growth in the presence of citric acid (right part in the Fig. 1). The as-prepared 3D hierarchical Ag-nanoplates decorated PAN nanopillar (denoted as AgNPs@PAN-nanopillar) arrays have the following advantages. First, the large-area highly ordered arrays of the PAN-nanopillar arrays can ensure the uniformity and reproducibility of SERS signals. Second, the densely packed $\mathrm{Ag}$ nanoplates could be assembled onto the 3D PAN-nanopillar-frameworks via a low cost and simple galvanic-cell-induced growth, and thus highly concentrated electromagnetic fields could be generated near the nanoscale sharp edges of the nanoplates and abundant uniform hot spots could also be achieved in the nano-gaps between the neighboring nanoplates in the hierarchical AgNPs@PAN-nanopillar arrays. Furthermore, using the hierarchical Ag-NPs@PAN-nanopillar arrays as sensitive SERSsubstrates, R6G, methyl parathion (one of extremely hazardous pesticides ${ }^{35}$ ) and PCB-77 (one congener of polychlorinated biphenyls belonging to persistent organic pollutants) with a concentration down to $10^{-11} \mathrm{M}, 10^{-7} \mathrm{M}$ and $10^{-6} \mathrm{M}$ can be recognized respectively, showing promising potentials in SERS-based rapid detection of toxic organic pollutants in the environment.

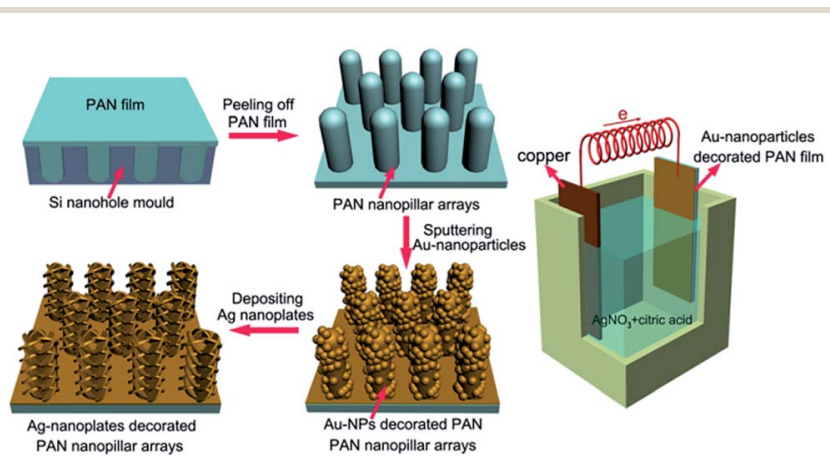

Fig. 1 Schematic for the fabrication of Ag-nanoplates-assembled PAN-nanopillar arrays via simple galvanic cell reaction method.

\section{Results and discussion}

\section{Fabrication of Ag-NPs@PAN-nanopillar arrays}

Fig. 2a and b show the oblique-view scanning electron microscope (SEM) images of the molded uniform PAN-nanopillar arrays on one side, revealing highly ordered hexagonal arrays of nanopillars with diameters of $\sim 268 \mathrm{~nm}$ and center-to-center distances of $\sim 600 \mathrm{~nm}$ (Fig. 2b). The enlarged oblique-view SEM image also reveals the arrays of PAN-nanopillars with heights of $\sim 550 \mathrm{~nm}$ (Fig. 2b). After ion-sputtering Au-nanoparticles onto the surface of the PAN-nanopillars as a conducting layer, dense Ag-nanoplates (Fig. 2c and d) were assembled onto the PANnanopillars via a simple galvanic cell reaction growth ${ }^{34}$ in a mixed electrolyte of $\mathrm{AgNO}_{3}$ and citric acid. After the galvanic cell reaction proceeding for $15 \mathrm{~min}$, the diameter and height of the nanopillars are $\sim 485 \mathrm{~nm}$ (Fig. $2 \mathrm{~d}$ ) and $\sim 670 \mathrm{~nm}$ (Fig. S1a and $\mathrm{b}$ of the ESI $\dagger$ ), respectively. The enlarged oblique-view and side-view SEM images (Fig. 2d, S1a and b of the ESI $\dagger$ ) reveal that cross-linked Ag-nanoplates were uniformly and densely decorated on both the top and the side surfaces of each PANnanopillar and high density sub-10 nm gaps are formed between the nearest Ag-nanoplates. Accordingly, the arrays of Ag-nanoplates decorated PAN-nanopillar possess high-density 3D "hot spots" due to strong coupling of such neighboring Ag-nanoplates. Energy dispersive X-ray spectroscopy (EDS) measurement (Fig. S2 of the ESI $\dagger$ ) confirms that the nanoplates are composed of Ag. Transmission electron microscopy (TEM)

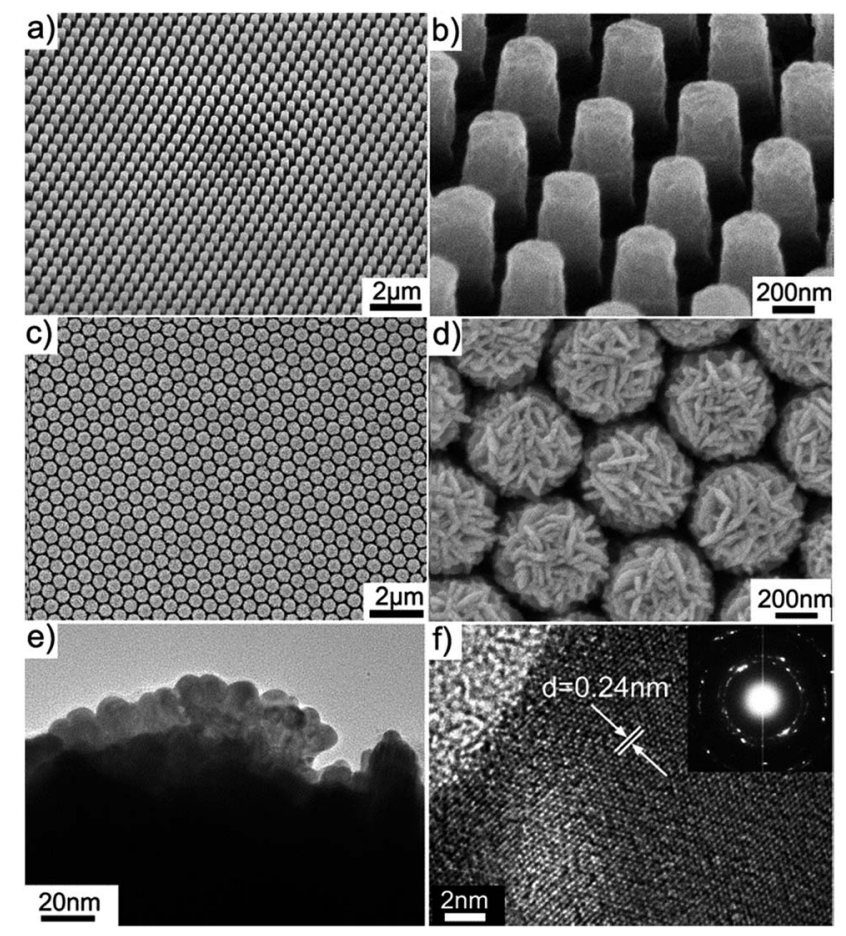

Fig. 2 ( $a$ and b) SEM images of the as-prepared hexagonal PANnanopillar arrays. (c and d) SEM images of Ag-nanoplates-assembled PAN-nanopillars. (e) A close-up TEM view of Ag-nanoplates assembled on one PAN-nanopillar. (f) HRTEM image of a single Ag nanoplate. The inset in $(f)$ is the corresponding selected area electron diffraction (SAED) pattern. 
and selected area electron diffraction (SAED) provide further insight into the structure of the Ag-nanoplates. The corresponding close-up TEM view of the products (Fig. 2c) shows the Ag-nanoplates are grafted on the surface of PAN-nanopillars (Fig. 2e), and the lattice-resolved image (Fig. 2f) taken on a single Ag-nanoplate reveals fringes with separated spacing of $2.4 \AA$, being assigned to the $\mathrm{Ag}\{111\}$ reflection. The inset in Fig. $2 \mathrm{f}$ is a typical SAED pattern taken from this nanoplate, showing the polycrystalline electron diffraction feature. The structure of the Ag-NPs@PAN-nanopillar arrays with different deposition durations is also verified by X-ray diffraction (XRD) pattern (Fig. S3 of the ESI $\dagger$ ). With prolongation of the $\mathrm{Ag}$ deposition duration to $45 \mathrm{~min}$, the corresponding XRD pattern shows four distinguishing diffraction peaks at 38.3, 44.4, 64.4, and $77.6^{\circ}$, corresponding to the (111), (200), (220), and (311) planes of the face-centered cubic (fcc) structure of silver, respectively.

\section{Morphology regulation of Ag-nanostructures wrapped PAN- nanopillar arrays}

The influence of PAN-nanopillar arrangement patterns. PANnanopillar arrays with different arrangement patterns and aspect ratios can also be used to fabricate Ag-NPs@PANnanopillar arrays via the facile galvanic-cell-induced growth. SEM images of highly ordered arrays of Ag-nanoplate assembled PAN-nanopillars with different arrangement patterns are shown in Fig. 3 and S4-S6 of the ESI. $\dagger$ Fig. 3a-c show tetragonal arrays of Ag-NPs@PAN-nanopillars with diameters of $\sim 280 \mathrm{~nm}$, and center-to-center distances of $\sim 330 \mathrm{~nm}$. And the enlarged topview SEM image (Fig. 3c) reveals that the surface roughness of

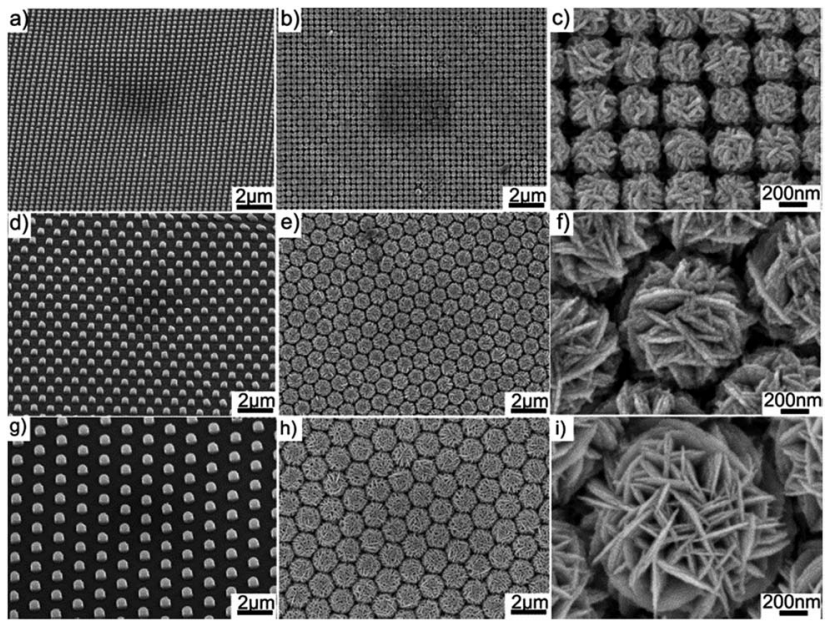

Fig. 3 (a) SEM image of tetragonal PAN-nanopillar arrays. (b and c) SEM images of tetragonal Ag-NPs@PAN-nanopillar arrays using the corresponding tetragonal PAN-nanopillar arrays shown in (a). (d) SEM image of the hexagonal PAN-nanopillar arrays (period: $1000 \mathrm{~nm}$ ). (e and f) SEM images of hexagonal Ag-NPs@PAN-nanopillar arrays using the corresponding hexagonal PAN-nanopillar arrays shown in (d). (g) SEM image of the hexagonal PAN-nanopillar arrays (period: $1500 \mathrm{~nm}$ ). ( $h$ and i) SEM images of hexagonal Ag-NPs@PAN-nanopillar arrays using the corresponding hexagonal PAN-nanopillar arrays shown in (g). the Ag-nanoplates decorated on the tetragonal PAN-nanopillar arrays is higher than that shown in the Ag-NPs@PANnanopillar arrays with hexagonal arrangement patterns (Fig. 2d). Such Ag-nanoplate assembled structures also contain many sub-10 nm gaps between the adjacent nanoplates (Fig. 3c and S4 of the ESI $\dagger$ ). It is worth mentioning that the hexagonal spherical Ag-nanoplate assembled structures (Fig. 3d-i, S5 and S6 of the ESI $\dagger$ ) were realized using the hexagonal PANnanopillar arrays as the deposition substrates due to the large spacing between the neighboring PAN-nanopillars. From the above results, the electrochemical growth mechanism of Agnanoplates decorated PAN-nanopillar arrays can be explained easily. Ag atoms or clusters are firstly generated at the projecting sites (Au-NPs-sputtered PAN nanopillars) by the reduction of $\mathrm{Ag}^{+}$via simple galvanic cell reaction, and they will nucleate on the Au-NPs-sputtered PAN nanopillars via heterogeneous nucleation, and then the nuclei grow into $\mathrm{Ag}$ nanostructures with the galvanic cell reaction going on. At the same time, the $\mathrm{Ag}$ nuclei formed on the Au-NPs will grow with the terminal planes $\{111\}$ with the lowest surface energy with the help of the citric acid acting as the capping agent and finally Ag nanoplates are achieved. As the "projecting sites" or "nuclear sites" on the PAN film can be regulated, therefore different geometric features of Ag-nanoplate wrapped structures can be easily controlled due to the selective nucleation sites on the pillars, which are determined by PAN-nanopillar arrays with different arrangement patterns, and all these Ag-NPs@PAN-nanopillar arrays show a high uniformity.

The influence of the concentration of citric acid. It has been demonstrated that citric acid has a great impact on the growth of the Ag-nanoplates. ${ }^{36}$ Fig. S7 of the ESI $\dagger$ shows the Agnanostructures wrapped PAN-nanopillar arrays prepared under the citric acid concentration of $0.5,2,4,8$ and $16 \mathrm{~g} \mathrm{~L}^{-1}$, respectively. When a low concentration of citric acid $\left(0.5 \mathrm{~g} \mathrm{~L}^{-1}\right)$ is added into the electrolyte, only small $\mathrm{Ag}$ nanoparticles can be deposited onto the surface of the PAN-nanopillars, as shown in Fig. S7a-d of the ESI. $\uparrow$ With the increase of citric acid concentration from $2 \mathrm{~g} \mathrm{~L}^{-1}$ to $8 \mathrm{~g} \mathrm{~L}^{-1}$, the morphology of the nanobuilding block on the PAN-nanopillar arrays evolves from quasi plate-like nanoparticle to plate-like nanostructure, and the size of Ag-nanoplate on the PAN-nanopillars become bigger and bigger as shown in Fig. S7e-p of the ESI. $\dagger$ In the meantime, with the growth of the Ag-nanoplates, the gaps between the neighboring nanopillars become narrower and narrower. If the concentration of citric acid is further increased, i.e. $16 \mathrm{~g} \mathrm{~L}^{-1}, \mathrm{Ag}$ NPs@PAN-nanopillars in the arrays are crosslinked with each other due to the confined growth space, rather than isolated Agnanoplate assembled nanopillars could be achieved (Fig. S7s and $t$ of the ESI $\dagger$ ). From the above results, it can be concluded that the morphology of $\mathrm{Ag}$ nanostructures grown on PANnanopillar arrays can be regulated by controlling the amount of the citric acid via the galvanic cell reaction growth. It was proved that citric acid could be used as a capping agent for the selective growth of Ag-nanoplates as it can selectively bind to $\{111\}$ facets. ${ }^{37}$ Here we suggest that citric acid also acts as a nucleation inhibitor besides the capping agent, inhibiting formation of new nuclei on the surface of the PAN-nanopillars. 
With the increase of the citric acid concentration, more citrate could be adsorbed on the PAN-nanopillars, thus the nucleation sites decrease on the surface for the reduction of $\mathrm{Ag}$ ions to $\mathrm{Ag}$ nanoparticles. Consequently, the number of the Ag-nanoplates decreases and the size increases with the raise of the citric acid concentration.

Structurally enhanced SERS performance. It is well known that the SERS performance of noble metal (especially Au and Ag) nanostructure arrays depends on both the size of the building blocks and the spacing between the neighboring assembly units. To optimize the SERS activity of the Ag-NPs@PANnanopillar arrays, Ag-deposition with different durations from 2 to $45 \mathrm{~min}$ was performed. The morphology evolution with the different deposition duration is shown in Fig. 4. It can be seen that the morphology of the Ag-nanostructures grafted PANnanopillar arrays could be efficiently regulated by the deposition duration. For short deposition duration $(2 \mathrm{~min})$, only irregularly shaped $\mathrm{Ag}$ nanoparticles are assembled onto the PAN-nanopillars (Fig. 4a). With the prolongation of the deposition, the number and size of the Ag-NPs gradually increase, as shown in Fig. $4 \mathrm{~b}$ for the deposition durations of $10 \mathrm{~min}$. When the deposition duration prolongs to 20-30 $\mathrm{min}$, the whole surface of the PAN-nanopillar are uniformly covered with crosslinked Ag-nanoplates as shown in Fig. 4c-e, and the spacing of the neighboring Ag-NPs@PAN-nanopillar gradually decreases. SEM images (Fig. 4d and e) show that three types of nanogaps can be achieved in the Ag-nanoplates decorated PAN-nanopillar arrays, i.e., nanogaps between the cross-linked Ag-NPs on the top ends of the same nanopillars, nanogaps between the crosslinked Ag-NPs on the sidewalls of the same nanopillars, and nanogaps between the Ag-NPs located on the adjacent nanopillars. Once the deposition is prolonged to $45 \mathrm{~min}$, the vertically aligned Ag-nanoplates coated on the PAN-nanopillars are connected with each other, thus Ag-nanoplate assembled films (Fig. 4f) were achieved. The corresponding absorption spectra of the resultant Ag-nanostructures grafted PAN-nanopillar arrays prepared under the different deposition durations were shown in Fig. $4 \mathrm{~g}$, which clearly approve that the morphology of nanostructure has prominent impact on the optical properties. The photographs show the distinct reflectance color from a dark red to a silver gray over a large area $(20 \mathrm{~mm} \times 20 \mathrm{~mm})$ due to the different absorption properties (insets of the Fig. 4). Strong plasmon coupling between the neighboring Ag-nanostructures grafted PAN-nanopillars are observed as the inter-spacing is decreased when the deposition duration is increased from $2 \mathrm{~min}$ to $45 \mathrm{~min}$ (Fig. 4g). The SERS activity of the corresponding as-fabricated Ag-nanostructures grafted PAN-nanopillars arrays was studied by using $10^{-7} \mathrm{M}$ rhodamine 6G (R6G) as probe molecules. Fig. 4h shows the SERS spectra of R6G collected on these $\mathrm{Ag}$ nanostructures grafted PAN-nanopillars array substrates achieved at the same citric acid concentration (4 $\mathrm{g} \mathrm{L}^{-1}$ ) but different Ag-deposition durations (from 2 to $45 \mathrm{~min}$ ). It can be found that the intensity of the Raman peaks increased obviously with increase of the $\mathrm{Ag}$ deposition duration from 2 to $30 \mathrm{~min}$, but decreased with the further elongation of Ag-deposition duration (Fig. 4h). As the Ag-deposition duration

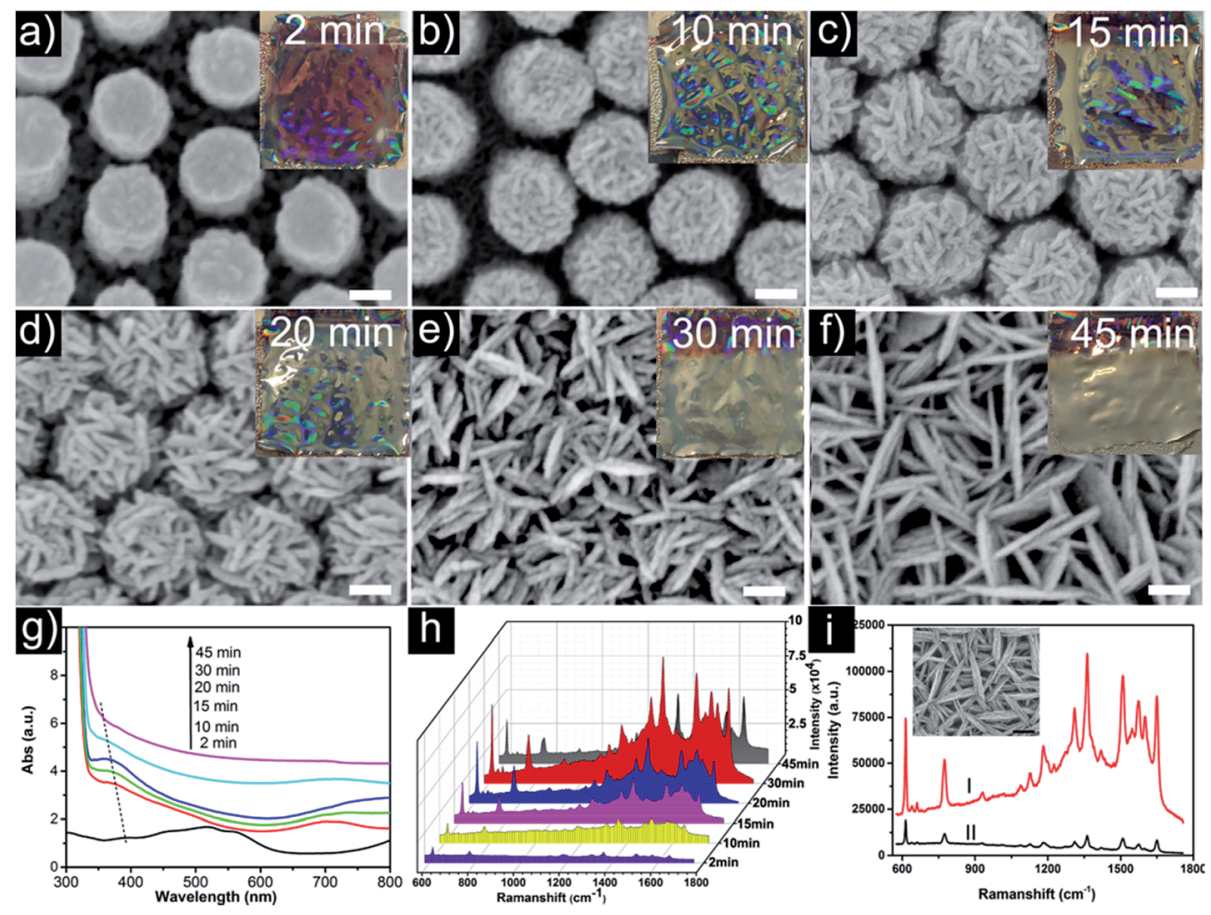

Fig. 4 (a-f) SEM images of the Ag-nanoshests-assembled PAN-nanopillar arrays with different Ag-depositing durations. Inset: the photographs of Ag-nanoplates assembled PAN-nanopillar films with different Ag-deposition durations scale bars: $200 \mathrm{~nm}$. (g) The corresponding UV-vis absorption spectra of Ag-NPs@PAN-nanopillar arrays prepared under the different Ag-depositing durations. (h) The SERS spectra of R6G collected on Ag-NPs@PAN-nanopillar arrays with different Ag-depositing durations. (i) The SERS spectra of R6G collected on the Ag-NPs@PANnanopillar arrays (curve I) and Ag-nanoplates assembled on the flat PAN film using electrochemical method (curve II); inset is SEM image of the Ag-nanoplates assembled on the flat PAN film using electrochemical method. Scale bars: $500 \mathrm{~nm}$. 
gradually increased from $2 \mathrm{~min}$ to $30 \mathrm{~min}$, larger number of $\mathrm{Ag}$ nanoplates could be generated, thus the nanogaps between the adjacent Ag-nanoplates on the single PAN-nanopillar increased correspondingly. Simultaneously, as the inter-spacing between two adjacent Ag-nanostructures grafted PAN-nanopillars is decreased gradually, the nanoscaled gaps could be formed between the top Ag-nanoplates located on the neighboring nanopillar resulting in an increase of another type of hot spots that provide a giant localized electromagnetic enhancement. ${ }^{15,17,28}$ In order to confirm the enhancement of SERS effect induced by the nanoscaled gaps between two adjacent Ag nanoplates grafted on different PAN-nanopillars, the SERS activity of the Ag nanoplates created on the flat PAN flim using electrochemical method was checked. The test results show that the Ag-nanoplates grafted PAN-nanopillars arrays show higher SERS activity than the Ag nanoplates created on the flat PAN flim (Fig. 4i). The comparison demonstrated that the 3D hierarchically Ag-nanoplates grafted PAN arrays show structurally enhanced SERS activity. When the Ag-deposition duration was prolonged to the $45 \mathrm{~min}$, Ag-nanoplates become larger and begin to connect together (Fig. 4f), resulting in the decrease of the nanoscale gaps between the neighboring Ag-nanoplates located on the neighboring nanopillar, thus the SERS activity deteriorates (Fig. 4h).

Obviously, the Ag-NPs@PAN-nanopillar arrays with $30 \mathrm{~min}$ $\mathrm{Ag}$ deposition duration exhibit the highest SERS activity, were thus chosen as SERS substrates to investigate the SERS signal sensitivity using R6G as probe molecule. Fig. 5a shows the Raman spectra of R6G with different concentrations $\left(10^{-8}\right.$, $10^{-9}, 10^{-10}$, and $10^{-11} \mathrm{M}$ ) adsorbed on the Ag-NPs@PANnanopillar arrays, displaying high quality SERS signals. As shown in Fig. 5a, the distinct bands can be still easily distinguished in the Raman spectrum even at the low concentration of $10^{-11} \mathrm{M}$. The SERS spectra reveal the characteristic peaks of
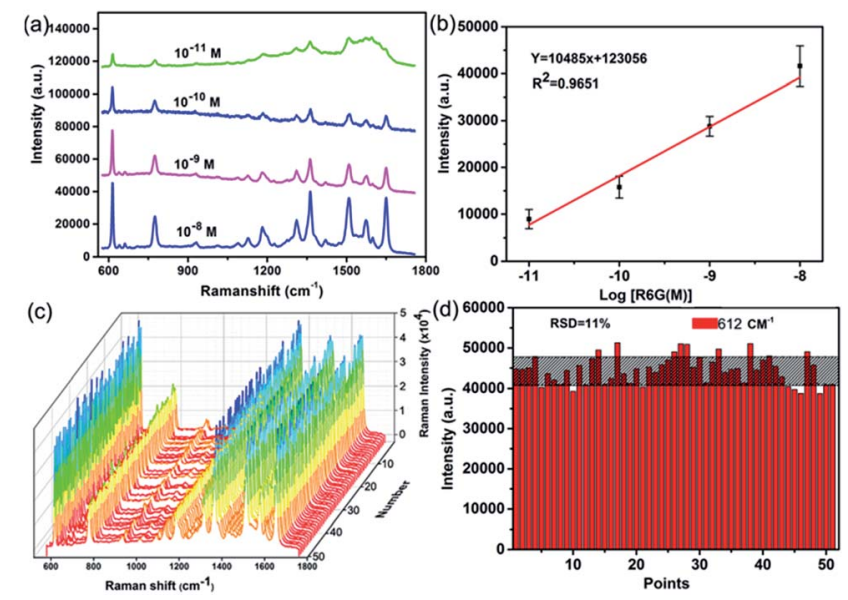

Fig. 5 (a) SERS spectra collected on the Ag-NPs@PAN-nanopillar arrays (Fig. 4e) exposed to different concentrations of R6G aqueous solution. (b) The corresponding calibration curve for Raman enhancement (at $612 \mathrm{~cm}^{-1}$ ) versus $\log [R 6 G(M)]$. (c) SERS spectra of $10^{-8} \mathrm{M}$ R6G collected from 50 random spots on the substrate shown in Fig. 4e. (d) The intensity of the peaks at $612 \mathrm{~cm}^{-1}$ from random 50 spots on the substrate.
R6G at $612 \mathrm{~cm}^{-1}$ (C-C-C ring in-plane bending mode), $775 \mathrm{~cm}^{-1}$ (C-C-C ring out-of-plane bending mode) and $1361 \mathrm{~cm}^{-1}$ (aromatic $\mathrm{C}-\mathrm{C}$ stretching vibration mode), respectively. ${ }^{32}$ The error bar line in Fig. 5b exhibits a correlation coefficient $R^{2}=0.96$ at the range of $10^{-11} \mathrm{M}$ to $10^{-8} \mathrm{M}$. The high SERS activity of the hierarchical SERS substrate is also demonstrated by a rough estimation of the enhancement factors (EF) shown in the part S2 of the ESI, $\uparrow$ where the 4-aminothiophenol (4-ATP) was used as the probe molecules. The EF can be calculated by the formula $\mathrm{EF}=\left(I_{\text {SERS }} / N_{\text {SERS }}\right) /\left(I_{\text {Ref }} / N_{\text {Ref }}\right)$, where $I_{\text {SERS }}$ and $I_{\text {Ref }}$ represent the relative peak intensities of the SERS spectra and the Raman spectra (non-SERS) respectively; and $N_{\text {SERS }}$ and $N_{\text {Ref }}$ represent the number of probe molecules on the substrates within the laser spot, respectively. The average EF is estimated to be about $9.6 \times 10^{6}$, showing the high SERS activity of the hierarchical SERS substrate.

For SERS-based applications, one of the crucial factors is the reproducibility of Raman signals from SERS substrate. The SERS signal reproducibility was investigated by taking the SERS spectra of R6G from different locations. Fig. 5c shows the SERS spectra of R6G collected at fifty spots on the substrate (Fig. 4e), the average relative standard deviation (RSD) of the intensities of the peaks $612 \mathrm{~cm}^{-1}$ is less than $12 \%$ (Fig. $5 \mathrm{~d}$ ), demonstrating a comparable uniformity of the SERS signal across the entire area of the substrate due to the ordered morphological features of the Ag-NPs@PAN-nanopillar arrays. The homogeneity of Ag nanoplate-assembled PAN-nanoarrays is further supported by the SERS images of "hot spots" over the large area. Fig. S8 of the ESI $\dagger$ shows the image for the arrays acquired with a spatial resolution of $1 \mu \mathrm{M}$ and presented as a color-coded integrated intensity of the characteristic peak of $\mathrm{R} 6 \mathrm{G}$ at $612 \mathrm{~cm}^{-1}$. It is obviously seen that the "hot spots" over the Ag nanoplateassembled PAN-nanopillar arrays are uniform.

To demonstrate potential applications of the as-prepared hierarchical SERS-substrates, the SERS-sensitivity of the AgNPs@PAN-nanopillar arrays to methyl parathion (a commonly used organophosphorus pesticide) and PCBs (one class of persistent organic pollutants, defined in the Stockholm Convention) was examined. Fig. 6 shows the SERS spectra of

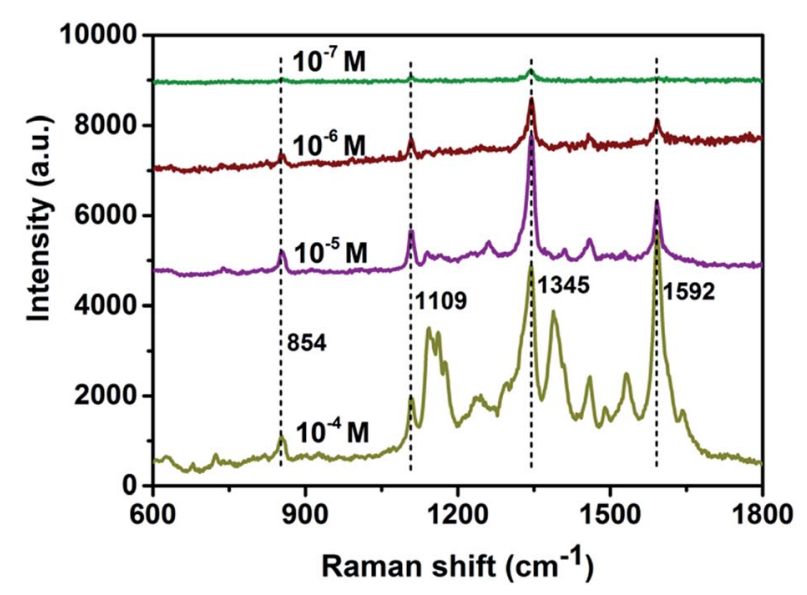

Fig. 6 SERS spectra of $10^{-4}, 10^{-5}, 10^{-6}$ and $10^{-7} \mathrm{M}$ methyl parathion probed using the substrate shown in Fig. $4 \mathrm{e}$. 
methyl parathion with different concentrations $\left(10^{-4}, 10^{-5}\right.$, $10^{-6}, 10^{-7} \mathrm{M}$ ) dispersed on the optimal hierarchical AgNPs@PAN-nanopillar arrays. It demonstrates that with a low concentration even down to $10^{-7} \mathrm{M}$, the characteristic peaks of $854,1109,1251,1345$ and $1592 \mathrm{~cm}^{-1}$ of methyl parathion can still be identified. ${ }^{38}$ Moreover, PCB-77 (one congener of PCBs) with different concentrations $\left(10^{-3}, 10^{-4}, 10^{-5}\right.$ and $\left.10^{-6} \mathrm{M}\right)$ have been detected, as shown in Fig. S9 of the ESI. $\dagger$ Five fingerprint characteristic peaks at 677, 1030, 1244, 1296 and $1596 \mathrm{~cm}^{-1}$ in the SERS spectra (Fig. S9†) correspond well to the reported results $^{39}$ and can still be recognized when the concentration of PCB-77 is down to $10^{-6} \mathrm{M}$.

\section{Conclusions}

In conclusion, large-scale SERS-active substrates consisting of Ag-nanoplate grafted PAN-nanopillar arrays have been achieved via a low-cost and green synthetic method. The morphology of Ag-NPs@PAN-nanopillar arrays could be regulated by controlling the Si molds with different arrangement patterns, the amount of the citric acid and Ag-deposition duration. The uniform SERS substrates possess both abundant 'hot spots' formed between the adjacent Ag-nanoplates, which ensure their excellent SERS-activity and signal repeatability. The AgNPs@PAN-nanopillar arrays have been used as SERS substrates for the detection, $10^{-11} \mathrm{M}$ R6G, $10^{-7} \mathrm{M}$ methyl parathion, and $10^{-6} \mathrm{M}$ PCB-77 are identified, showing promising potentials in rapid recognition of chemical pollutants.

\section{Experimental section}

\section{Materials}

Polyacrylonitrile (PAN, $M_{\mathrm{w}} 150$ 000) was purchased from sigmaAldrich. Dimethyl formamide (DMF), ethanol (AR), rhodamine 6G (R6G) and methyl parathion were purchased from Shanghai Aladdin Reagent Co. Ltd. (Shanghai, China). PCB-77 was purchased from AccuStandard Inc. The water used in all experiments was ultrapure $(18.2 \mathrm{M} \Omega)$. All chemicals were used as received without further purification.

\section{Preparation of PAN-nanopillar arrays}

200-300 $\mu \mathrm{L}$ of as-prepared $8 \mathrm{wt} \%$ of PAN DMF solution was casted onto the nanohole array Si mold uniformly. The mold was heated at $80^{\circ} \mathrm{C}$ for 15 min to evaporate off the DMF solvent. Then PAN-nanopillar arrays can be achieved and directly transferred onto any substrate.

\section{Sputtering Au-nanoparticles onto the PAN-nanopillar arrays}

Before sputtering the Au-nanoparticles onto the surfaces of the PAN-nanopillar arrays, the as-prepared PAN-nanopillar arrays were fixed on flat glass wafers. Au-nanoparticles were sputtered on the PAN-nanopillar arrays using a K550X sputter coater with an electric current of $40 \mathrm{~mA}$ for $3 \mathrm{~min}$.

\section{Preparation of Ag-NPs@PAN-nanopillar arrays via simple} galvanic-cell-induced growth method

$0.06 \mathrm{~g} \mathrm{AgNO}_{3}$ and $0.12 \mathrm{~g}$ citric acid were added to $30 \mathrm{~mL} \mathrm{DI}$ water, followed by stirring until complete dissolution of the solute. The resulting homogeneous solution used as the electrolyte in the experiment was transferred to a galvanic cell. A piece of copper foil $(1.5 \mathrm{~cm} \times 1.5 \mathrm{~cm})$ connected to the Aunanoparticles-sputtered PAN-nanopillar arrays with a conducting wire used as the anode. After the deposition for $15 \mathrm{~min}$ at room temperature, the PAN-nanopillar arrays with the products were then taken out, cleaned with DI water several times and dried with high-purity flowing nitrogen.

\section{Characterization}

The resultant products were characterized by using X-ray diffraction (XRD) (Philips X'pert-PRO), scanning electronic microscope (SEM, sirion 200) with energy-dispersive X-ray spectroscopy (EDS, OXFORD) and Ultraviolet-Visible-NearInfrared spectrophotometer (Hitachi, U-4100).

\section{SERS measurements}

SERS measurements for R6G, polychlorinated biphenyls (PCBs) and methyl parathion were performed with the Renishaw inVia microscope equipped with Leica microscope $(50 \times$ objective $)$. For the purpose of good molecule adsorption, the AgNPs@PAN-nanopillar array substrates were immersed in $1 \mathrm{~mL}$ R6G aqueous solution with different concentrations (from $10^{-7}$ to $10^{-11} \mathrm{M}$ ) for 3 hours, taken out and then dried with highpurity flowing nitrogen. For the purpose of good molecule adsorption, the substrates were immersed in methyl parathion solution with different concentrations and PCB-77 $n$-hexane solutions with different concentrations for 5 hours, taken out and then dried with high-purity flowing nitrogen. SERS measurements were carried out under a confocal microprobe Raman system (Renishaw, inVia) with an excitation wavelength of $532 \mathrm{~nm}$. The exposure times for R6G, methyl parathion and PCB-77 were $5 \mathrm{~s}, 10 \mathrm{~s}$ and $30 \mathrm{~s}$.

Raman mappings of "hot spots" over Ag-NPs@PANnanopillar arrays were performed on Raman imaging system (Renishaw, inVia). R6G $\left(10^{-8} \mathrm{M}\right)$ was used as molecular probe. A $532 \mathrm{~nm}$ laser was scanned over the substrates and Raman spectra were collected as a function of position. Renishaw WiRE 3.2 software was used to data acquisition and control.

\section{Conflicts of interest}

There are no conflicts to declare.

\section{Acknowledgements}

This work was supported by National Key Basic Research Program of China (Grant No. 2013CB934304), NSFC (51501183), Talent Project of Anhui Agriculture University (yj2017-12). 


\section{Notes and references}

1 D. Graham and R. Goodacre, Chem. Soc. Rev., 2008, 37, 883884.

2 R. A. Tripp, R. A. Dluhy and Y. Zhao, Nano Today, 2008, 3, 3137.

3 J. F. Li, Y. F. Huang, Y. Ding, Z. L. Yang, S. B. Li, X. S. Zhou, F. R. Fan, W. Zhang, Z. Y. Zhou, Y. WuDe, B. Ren, Z. L. Wang and Z. Q. Tian, Nature, 2010, 464, 392-395.

4 K. V. Kong, Z. Lam, W. D. Goh, W. K. Leong and M. Olivo, Angew. Chem., 2012, 124, 9934-9937.

5 K. Saha, S. S. Agasti, C. Kim, X. Li and V. M. Rotello, Chem. Rev., 2012, 112, 2739-2779.

6 J. Ando, M. Asanuma, K. Dodo, H. Yamakoshi, S. Kawata, K. Fujita and M. Sodeoka, J. Am. Chem. Soc., 2016, 138, 13901-13910.

7 L. Yang, P. Li and J. Liu, RSC Adv., 2014, 4, 49635-49646.

8 K. Zhao, C. Wu, Z. Deng, Y. Guo and B. Peng, RSC Adv., 2015, 5, 52726-52736.

9 M. J. Banholzer, J. E. Millstone, L. Qin and C. A. Mirkin, Chem. Soc. Rev., 2008, 37, 885-897.

10 B. Shang, Y. Wang, P. Yang, B. Peng and Z. Deng, Sens. Actuators, B, 2018, 255, 995-1005.

11 H. Im, K. C. Bantz, S. H. Lee, T. W. Johnson, C. L. Haynes and S.-H. Oh, Adv. Mater., 2013, 25, 2678-2685.

12 Y. Li, M. L. Simeral and D. Natelson, J. Phys. Chem. C, 2016, 120, 22558-22564.

13 Y. Lu, G. L. Liu, J. Kim, Y. X. Mejia and L. P. Lee, Nano Lett., 2005, 5, 119-124.

14 C. Zhu, G. Meng, Q. Huang, X. Wang, Y. Qian, X. Hu, H. Tang and N. Wu, Nano Res., 2015, 8, 957-966.

15 A. Kim, F. S. Ou, D. A. A. Ohlberg, M. Hu, R. S. Williams and Z. Li, J. Am. Chem. Soc., 2011, 133, 8234-8239.

16 C. Zhu, G. Meng, P. Zheng, Q. Huang, Z. Li, X. Hu, X. Wang, Z. Huang, F. Li and N. Wu, Adv. Mater., 2016, 28, 4871-4876.

17 Y. Feng, Y. Wang, H. Wang, T. Chen, Y. Y. Tay, L. Yao, Q. Yan, S. Li and H. Chen, Small, 2012, 8, 246-251.

18 J.-C. Valmalette, Z. Tan, H. Abe and S. Ohara, Sci. Rep., 2014, 4, 5238.

19 H. Wang, C. S. Levin and N. J. Halas, J. Am. Chem. Soc., 2005, 127, 14992-14993.
20 X. He, C. Yue, Y. Zang, J. Yin, S. Sun, J. Li and J. Kang, J. Mater. Chem. A, 2013, 1, 15010-15015.

21 S. Y. Lee, S.-H. Kim, M. P. Kim, H. C. Jeon, H. Kang, H. J. Kim, B. J. Kim and S.-M. Yang, Chem. Mater., 2013, 25, 2421-2426.

22 R. Kattumenu, C. H. Lee, L. Tian, M. E. McConney and S. Singamaneni, J. Mater. Chem., 2011, 21, 15218-15223.

23 S. Lee, M. G. Hahm, R. Vajtai, D. P. Hashim, T. Thurakitseree, A. C. Chipara, P. M. Ajayan and J. H. Hafner, Adv. Mater., 2012, 24, 5261-5266.

24 F. Jiang, S. Wang, L. Li, H. Jin, W. Zhang, J. Lin, T. Tang and J. Wang, Green Chem., 2011, 13, 2831-2836.

25 S. Jones, S. S. Sinha, A. Pramanik and P. C. Ray, Nanoscale, 2016, 8, 18301-18308.

26 Y.-J. Oh and K.-H. Jeong, Adv. Mater., 2012, 24, 2234-2237.

27 K. Zhao, J. Zhao, C. Wu, S. Zhang, Z. Deng, X. Hu, M. Chen and B. Peng, RSC Adv., 2015, 5, 69543-69554.

28 Z. Li, G. Meng, Q. Huang, X. Hu, X. He, H. Tang, Z. Wang and F. Li, Small, 2015, 11, 5452-5459.

29 F. Shao, Z. Lu, C. Liu, H. Han, K. Chen, W. Li, Q. He, H. Peng and J. Chen, ACS Appl. Mater. Interfaces, 2014, 6, 6281-6289.

30 M. S. Schmidt, J. Hübner and A. Boisen, Adv. Mater., 2012, 24, OP11-OP18.

31 X. Li, G. Chen, L. Yang, Z. Jin and J. Liu, Adv. Funct. Mater., 2010, 20, 2815-2824.

32 J. Liu, G. Meng, Z. Li, Z. Huang and X. Li, Nanoscale, 2015, 7, 18218-18224.

33 I. Pastoriza-Santos and L. M. Liz-Marzan, J. Mater. Chem., 2008, 18, 1724-1737.

34 Z. Li, G. Meng, Q. Huang, C. Zhu, Z. Zhang and X. Li, Chem.Eur. J., 2012, 18, 14948-14953.

35 G. K. Isbister, K. Mills, L. E. Friberg, M. Hodge, E. O'Connor, R. Patel, M. Abeyewardene and M. Eddleston, Clin. Toxicol., 2007, 45, 956-960.

36 H. Tang, G. Meng, Z. Li, C. Zhu, Z. Huang, Z. Wang and F. Li, Nano Res., 2015, 8, 2261-2270.

37 X. C. Jiang, C. Y. Chen, W. M. Chen and A. B. Yu, Langmuir, 2010, 26, 4400-4408.

38 D. Lee, S. Lee, G. H. Seong, J. Choo, E. K. Lee, D.-G. Gweon and S. Lee, Appl. Spectrosc., 2006, 60, 373-377.

39 Q. Zhou, Y. Yang, J. Ni, Z. Li and Z. Zhang, Nano Res., 2010, 3, 423-428. 